\title{
Malignant Rhabdoid Tumor of Brain: An Aggressive Clinical Entity
}

\author{
B.J. Fisher, J. Siddiqui, D. Macdonald, A.E. Cairney, D. Ramsey, D. Munoz and \\ R. Del Maestro
}

\begin{abstract}
Objective: We report three patients with malignant rhabdoid tumor (MRT) of the brain, two children and an adult. There were three purposes to this report: to describe the clinical course in an adult with MRT; to describe the interesting histopathological metamorphosis of one of the tumors; and to report the outcome of the treatment regimens we used in order to help guide future treatment. Since these tumors are quite rare it is important to continue to try new regimens in the search for effective therapy rather than to repeat ineffective ones. Method: Report of three patients. Results: The clinical course in all three patients was typical of these aggressive neoplasms in that chemotherapy and radiotherapy were ineffective in modifying the rapid deterioration leading to death. Conclusions: MRT can occur in adults. Autopsy in one patient showed that the tumor seemed to undergo an evolution in appearance when compared with the original pathology specimen from craniotomy. Administration of systemic therapy should be prompt and include intrathecal chemotherapy.
\end{abstract}

RÉSUMÉ: Tumeur rhabdoïde maligne du cerveau: une entité clinique agressive. Objectif: Nous rapportons les cas de trois patients atteints de tumeurs rhabdoïdes malignes (TRM) du cerveau, deux enfants et un adulte. Il y a trois buts à cette présentation: décrire l'évolution clinique chez un adulte avec une TRM; décrire la métamorphose histopathologique intéressante de l'une des tumeurs; rapporter les résultats du traitement afín de contribuer à améliorer le traitement de ces tumeurs. Comme ce sont des tumeurs qui sont rares, il est important d'essayer de nouveaux traitements dans notre recherche d'une thérapie efficace plutôt que de continuer à utiliser ceux qui ne sont pas efficaces. Méthodes: Il s'agit d'un compte rendu de trois cas. Résultats: L'évolution clinique chez les trois patients était typique de ces néoplasies agressives du fait que la chimiothérapie et la radiothérapie sont inefficaces et ne modifient pas la détérioration rapide conduisant au décès. Conclusions: La TRM peut survenir chez l'adulte. Chez un patient, l'autopsie a montré une évolution dans l'aspect de la tumeur par comparaison avec le spécimen anatomopathologique original obtenu par crâniotomie. L'administration d'une thérapie systémique devrait être prompte et inclure une chimiothérapie intrathécale.

Can. J. Neurol. Sci. 1996; 23: 257-263

Malignant rhabdoid tumors (MRT) are rare neoplasms which predominantly occur in infants and young children. The most frequent site of origin is the kidney. Extra-renal MRT's are much rarer. To date, 25 cases of primary CNS MRT have been reported (Table 1). ${ }^{1-17}$ These tumors behave in an extremely aggressive manner with rapid development of widespread metastases and therefore have an extremely poor prognosis. ${ }^{14,15}$ To date an effective therapeutic regimen remains elusive. We present three cases of primary MRT.

\section{Reports of Patients}

\section{Patient 1}

The patient was a ten-year-old girl. She became symptomatic in September 1992 with severe frontal headaches and vomiting. By January 1993, she began to complain of abdominal pain, blurring of vision and diplopia. On examination she was found to be irritable with a left homonymous hemianopsia, left sixth nerve palsy, bilateral papilledema and a mild right hyperreflexia. A CT scan of brain (Figure la) revealed an enhancing right occipital mass.
A craniotomy and gross total resection was performed in February 1993. Pathological diagnosis was malignant rhabdoid tumor. The tumor was seen to be invading into, but not arising, from central nervous system tissue. Light microscopy with hematoxylin and eosin (Figure 1b) stain revealed sheets and strands of medium to large epithelioid tumor cells with an extreme degree of pleomorphism and high mitotic rate but no obvious glandular, organoid or fibrillary differentiation. The tumor nuclei were vesicular with prominent nucleoli, often eccentrically located. Some of the larger cells contained large eosinophilic cytoplasmic inclusions which by electron microscopy consisted of loose fascicles of intermediate filaments with no evidence of skeletal muscle differentiation. There were no thick or thin filaments of $\mathrm{Z}$ bands. Immunohistochemical staining for

From the Department of Radiation Oncology, London Regional Cancer Centre (B.J.F.); Deparment of Neurosurgery (J.S., R.D.M.); Department of Neurology (D.M.); Department of Paediatrics (A.E.C.); Department of Pathology (D.R.. D.M.), London Health Sciences Centre, London.

RECEIVED JANUARY 2, 1996. ACCEPTED IN FINAL FORM MAY 16, 1996.

Reprint requests to: Dr. B. Fisher, London Regional Cancer Centre, 790 Commissioners Road East, London, Ontario, Canada N6A 4L6 
Table 1. Summary of Distribution, Management and Outcome of CNS MRT.

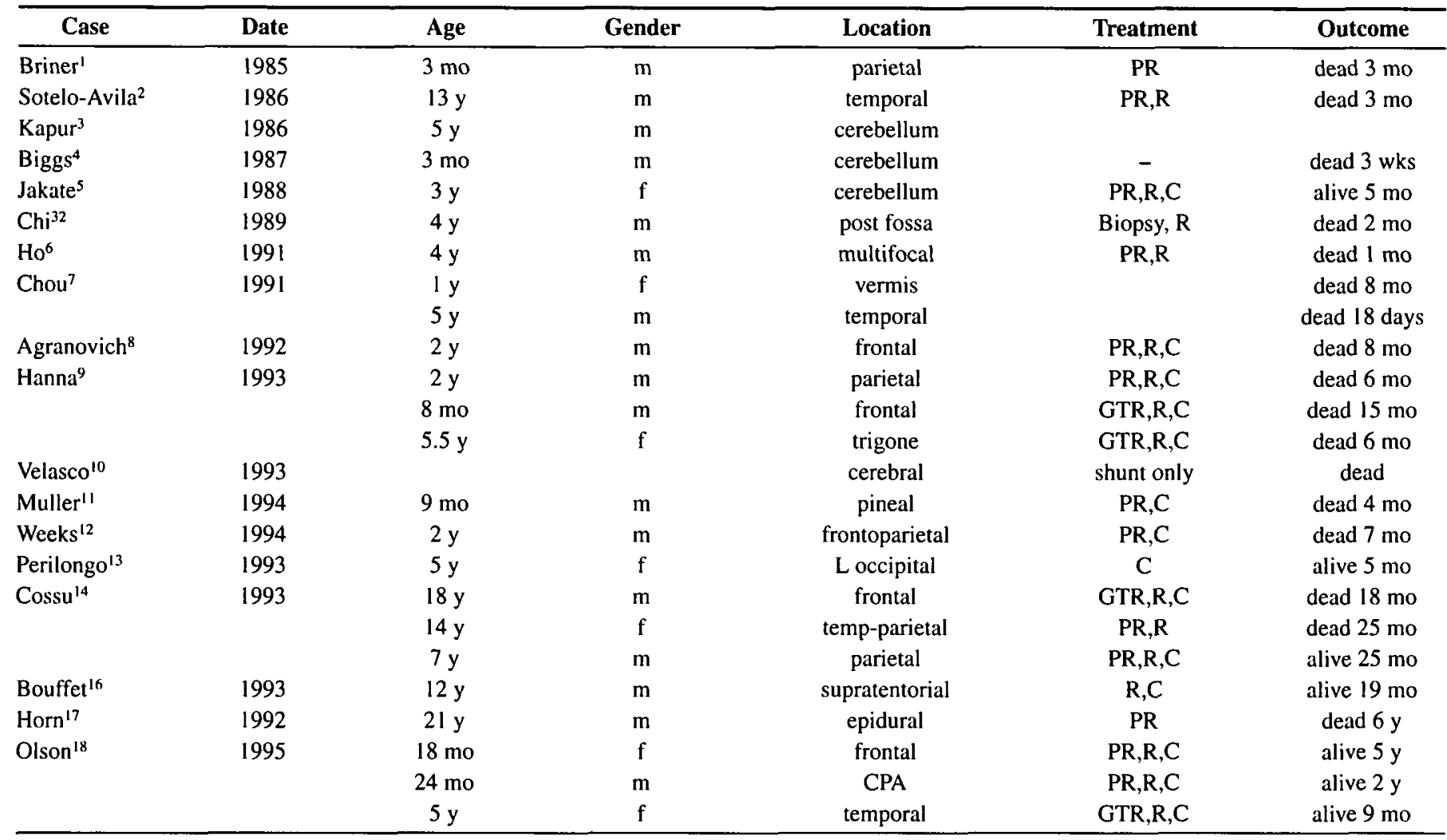

$\mathrm{PR}=$ Partial Resection, $\mathrm{R}=$ Radiotherapy, $\mathrm{C}=$ Chemotherapy

GTR $=$ Gross Total Resection, $\mathrm{M}=$ Male, $\mathrm{F}=$ Female
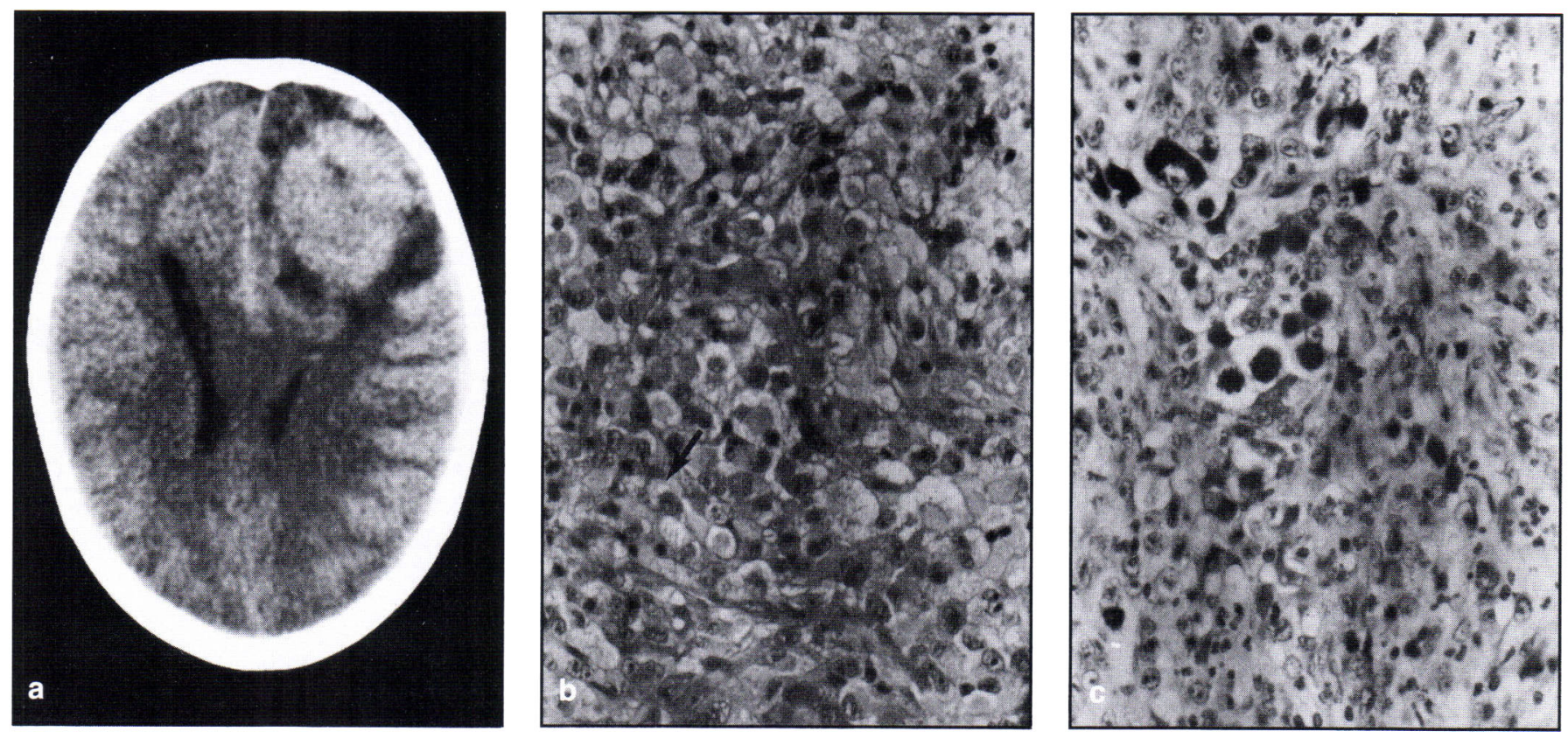

Figure 1: Patient 1. a) Preoperative MRI scan of brain. b) Hematoxylin and eosin photomicrograph from loosely arranged part of tumor. Note the large cells with cytoplasmic inclusions which displace the nucleus to one side. c) Vimentin stain. 
S-100 muscle specific antigen, actin, desmin and glial acidic protein (GFAP) were negative. There was weak cytokeratin positivity in the inclusions. Both tumor cells and inclusions were strongly vimentin (Figure 1c) and ubiquitin positive. Synaptophysin was positive in rare cell processes, but these could not be traced to obviously neoplastic cells. A moderate amount of glycogen was also present. The cells showed interdigitating cell processes and rare simple attachments, but no evidence of desmosomes or synaptic vesicles.

Postoperative workup included chest $x$-ray, CT scans of chest and abdomen, bone scan and MRI of spine, all of which were negative. Two weeks postsurgery the patient developed a recurrence of her symptomatology. CT scan of the brain revealed a contrast-enhancing mass at the original tumor site with surrounding edema. The radiological appearance was thought to be representative of recurrent tumor. Cerebrospinal fluid cytology was positive. The child commenced chemotherapy consisting of cyclophosphamide $\left(65 \mathrm{mg} / \mathrm{m}^{2} \mathrm{IV}\right)$, vincristine $\left(1.5 \mathrm{mg} / \mathrm{m}^{2} \mathrm{IV}\right)$ and mesna ( $250 \mathrm{mg} \mathrm{q} 3 \mathrm{~h} \times 8$ doses). Concurrently she began cranial irradiation by means of a lateral parallel pair of focal fields encompassing the recurrent tumor to 1800 cGy over 10 fractions using a combination of 6 and $18 \mathrm{MV}$ photons. She was to proceed with craniospinal radiation as soon as her blood counts recovered.

Two weeks later the child was readmitted to hospital with severe back pain. Repeat MRI of spine disclosed the presence of extensive thoracolumbar vertebral metastases, only three weeks after a negative MRI of spine. Palliative spinal radiotherapy was administered and the child expired two weeks later.

\section{Patient 2}

This previously healthy six-year-old child presented in April 1994 with a six week history of headache, irritability, insomnia, lethargy and occasional vomiting. She underwent a CT scan of brain in May 1994 (Figure 2a) which revealed a large left temporoparietal mass. An MRI confirmed a $4.6 \times 4.6 \mathrm{~cm}$ mixed signal intensity mass in the left parietal lobe and the trigone of the lateral ventricle. There was evidence of calcification, hemorrhage and contrast enhancement.

On May 20, 1994 the child underwent a craniotomy and subtotal excision of a necrotic tumor. Pathology reported malignant rhabdoid tumor. A postoperative lumbar puncture revealed elevated pressure so a lumbar drain was inserted. CSF cytology was positive. Other investigations included a bone marrow aspirate and biopsy, chest $x$-ray, ultrasound of the abdomen and pelvis, and CT scans of the chest, abdomen and pelvis, all of which were negative. Chemotherapy was initiated in June 1994 with vincristine $1.5 \mathrm{mg} / \mathrm{m}^{2}$ IV route days 1,8 and 15 , ifosfamide $3 \mathrm{~g} / \mathrm{m}^{2}$ IV days 1 and 2, carboplatin $500 \mathrm{mg} / \mathrm{m}^{2}$ IV day 3 , and etoposide $150 \mathrm{mg} / \mathrm{m}^{2}$ IV days 1 and 2 every 3 weeks.

Myelosuppression and sepsis occurred and were treated. A second cycle of chemotherapy using the same agents and doses was administered three weeks later.

Craniospinal radiation commenced in mid June 1994. The cranium was treated with parallel opposed lateral portals using $4 \mathrm{MV}$ photons, and the spine was treated with a single direct $16 \mathrm{MV}$ electron field. A dose of 3600 cGy over 20 fractions was prescribed to the craniospinal axis. A boost of an additional 1800 cGy over 10 fractions was administered using a weighted parallel opposed pair of 6 and $18 \mathrm{MV}$ photon fields. The craniospinal junction was moved weekly. There were a number of treatment interruptions because of excessive skin reactions and neutropenia attributed to the combination of chemotherapy and radiotherapy. Despite this aggressive treatment a repeat CT scan in early July 1994 reported an enlarging tumor with increased enhancement. The child died in October 1994 of respiratory failure. An autopsy restricted to the brain, spinal cord and abdomen was performed.

\section{Autopsy findings}

Gross examination of the brain revealed a well circumscribed yellow-green-brown $2 \mathrm{~cm}$ mass involving the left lateral ventricle. The corpus callosum was thinned and hydrocephalus was present. There was expansion and opacification of the meninges with multiple deposits encasing the spinal cord and meninges. A pale extramedullary mass covered the cauda equina.

The meninges were infiltrated by polygonal cells with a pleomorphic appearance. In areas of pronounced growth the cells were arranged in sheets with patchy areas of necrosis. In some cells the nucleus was pushed to one side by apparent cytoplasmic inclusions and mitotic figures.

The tumor mass itself consisted of sheets of cells abruptly interfacing with normal brain tissue at some sites and infiltrating it diffusely in other areas. In some areas the cells spilled into the ventricle. There were large areas of necrosis within the tumor. Areas adjacent to the tumor were edematous, with reactive astrocytosis suggestive of post radiation change. The intraparenchymal cells tended to be arranged in coarse fascicle spindle cells with striking, coarse processes (Figure 3a and b). Cells with cytoplasmic inclusions were distinctly rare. Their appearance was very different from cells from the craniotomy specimen (Figure $2 b$, c) and cerebrospinal fluid prior to chemoradiation and from the polygonal cells present in the meninges at autopsy. A focally prominent reticulin network was present. The immunohistochemistry findings were as follows: cytokeratin and muscle-specific actin negative, S-100 and vimentin strongly positive.

\section{Patient 3}

This 32-year-old gentleman presented in April 1995 with sudden onset of bifrontal headache, nausea and vomiting. Over the next one and a half weeks he developed progressive headaches, visual loss especially in the right eye and a $15 \mathrm{~kg}$ weight loss. He had been experiencing general malaise and weakness over the previous two months. CT scan of the brain (Figure $4 \mathrm{a}$ ) revealed a left periventricular caudate lesion. The patient did not improve with steroids.

A stereotactic biopsy was performed in July, 1995. Pathological diagnosis was malignant rhabdoid tumor. The tumor was composed of sheets of epithelioid cells with no evidence of epithelial architecture. Prominent nucleoli were often displaced to the periphery by cytoplasmic spherical masses displaying a faint fibrillarity. Numerous mitoses were identified (Figure 4b). Most tumor cells intensely expressed vimentin (Figure 4c) in their cytoplasm. Rare tumor cells expressed S-100. Immunohistochemical staining for LCA. cytokeratin L26 and cytokeratin was uniformly negative. Occasional cells displayed intense cytoplasmic immunoreactivity for lambda or kappa immunoglobulin light chain and cytoplasmic GFAP (glial fibrillary acidic protein) or PAS stain. The specimen displayed nonspecific staining for UCHL-1. Neoplastic cells were uniformly negative in Fontana stained sections and positive in Alcian blue stained sections.

A ventriculoperitoneal shunt was inserted to prevent progressive hydrocephalus. Other investigations performed included CBC, biochemistry, chest $x$-ray, bone scan and abdominal ultrasound which were al negative. MRl of the spine revealed evidence of leptomeningeal metastases. CSF glucose was reduced at $0.8 \mathrm{mMol} / \mathrm{L}$ and CSF protein was slightly elevated to $459 \mathrm{mMol} / \mathrm{L}$ : CSF cytology was reported to be suspicious for malignancy.

The patient remained drowsy, confused and agitated, with symptoms and signs of meningeal irritation. He was functionally blind. In view of the pathological diagnosis, its prognosis, the performance status of the patient and the lack of reported effective therapy, the patient was treated with symptomatic comfort measures only and he died in August 1995. 

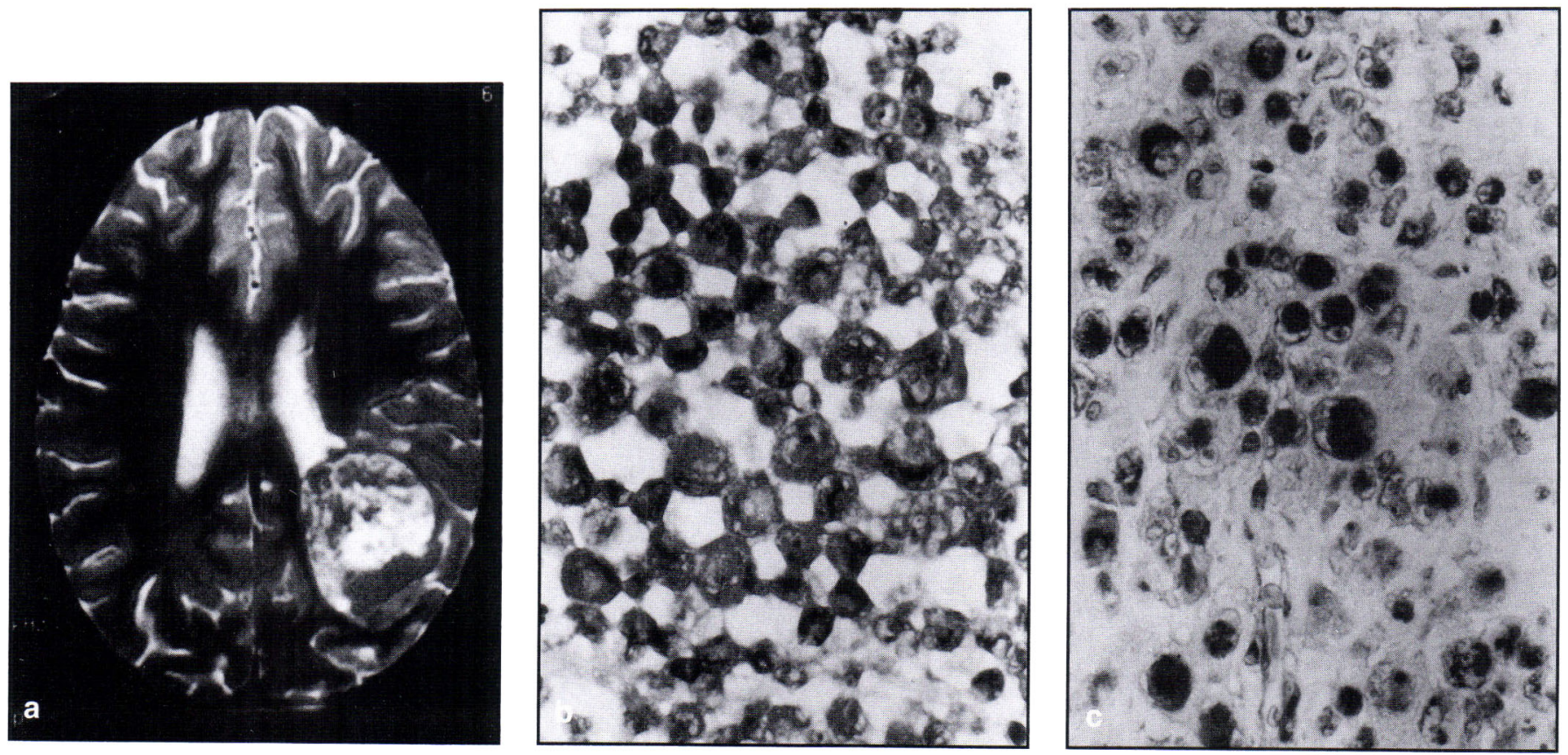

Figure 2: Patient 2. a) Preoperative MRI scan of brain. b) Photomicrograph of hematoxylin and eosin staining. c) Photomicrograph of vimentin staining.

\section{DISCUSSION}

Malignant rhabdoid tumor is a rare entity usually occurring in infants and young children but they can occur in adults as well, as seen in Patient 3. To date twenty-five cases of primary malignant rhabdoid tumor of the central nervous system have been described. ${ }^{1-18}$ Originally described as occurring in kidney, ${ }^{19,20}$ extrarenal primaries have since been identified in liver, ${ }^{21,22}$ soft tissue, ${ }^{23,24}$ thymus and central nervous system. ${ }^{1,4,5,8}$ The original description of MRT of the kidney was made during the First National Wilms' Tumor Study Group project in the United States. At that time some of the rapidly fatal tumors were noted to have a distinct monomorphic histology bearing a resemblance to rhabdomyosarcoma. ${ }^{22}$ The "rhabdoid" designation for this tumor has persisted although no evidence of differentiation into skeletal muscle has ever been found by electron microscopy or immunohistochemistry. The differentation between MRT and a more favourable diagnosis depends on the presence of filamentous inclusions and the absence of differentiation into other recognizable tissue elements. ${ }^{12}$

Briner et al. ${ }^{1}$ reported the first unequivocal case of primary CNS malignant rhabdoid tumor of brain in 1987; previous reports had failed to exclude the possibility that the intracranial tumor represented a metastasis from another site. That infant died a few weeks post-diagnosis and was found at autopsy to have a cerebellar mass and subarachnoid seeding but no other primary site was identified.

Pathologically CNS MRT's are characterized by distinct morphological and immunohistochemical features. The neoplastic cells are round or polygonal in shape with a diffuse growth pattern, moderate pleomorphism, high mitotic rate, a single nucleus and single macronucleolus. They are indistinguishable from their renal counterpart. The cells have an eosinophilic cytoplasm containing hyaline inclusions which on ultrastructural examination consist of whorls of intermediate filaments. Immunohistochemical stains for cytoplasmic neurofilament, myoglobulin and desmin are negative but stains for epithelial membrane antigen and vimentin are positive in most studies. $1.7,25$

Theories of histogenesis have implicated mesenchymal, histiocytic and neuroectodermal origin for MRT. ${ }^{26}$ An early suggestion of histiocytic orgin has not been supported. ${ }^{23}$ Weeks $^{12}$ theorized that these tumors were a form of PNET. Muller ${ }^{11}$ has stated that although the histogenesis of these tumors remains unknown, he favoured an origin from a primitive meningeopithelial cell because of the reported immunopositivity for vimentin, cytokeratin and epithelial membrane antigen in MRTs. The ubiquitin positivity in one of our cases suggests that the inclusions are more analogous to Mallory's hyaline inclusions (cytokeratin + ubiquitin) or Lewy body (neurofilament + ubiquitin). Ota ${ }^{27}$ performed phenotypic, cytogenetic and molecular analysis of two malignant rhabdoid cell lines. The cells demonstrated multiphenotypic neuroecto-mesenchymal differentiation with a single translocation at $+(11,12)$ (p 15.5:91-23). Variable expression of neural markers, and the association of renal MRT with medulloblastoma ${ }^{28}$ has led to the suggestion of $\mathrm{Ota}^{27}$ and others that MRT is a primitive neuroectodermal neoplasm arising from a primitive pluripotential cell of origin. Biegel ${ }^{29}$ reported two MRT's demonstrating monosomy of chromosome 22 . The relatively normal karyotype in these cases suggests that the aggressive phenotype of MRT may yet be understandable in molecular genetic terms and possibly reversible. Evidence to support this hypothesis has accidently been generated by researchers looking for a genetic basis for Wilms' tumor. Subclones of the "nephroblastoma" line ATCC G401 were used to demonstrate the presence of a tumor suppressor region on the short arm of chromosome 11.30. Subsequent reanalysis of the parent cell line revealed the typical histomorphology and immunostaining of MRT. ${ }^{31}$

It is interesting that in one of our patients (Patient 2) the tumor underwent a metamorphosis in light microscopic appearance between the time of craniotomy and autopsy. The surgical pathology specimen consisted of polygonal cells with 

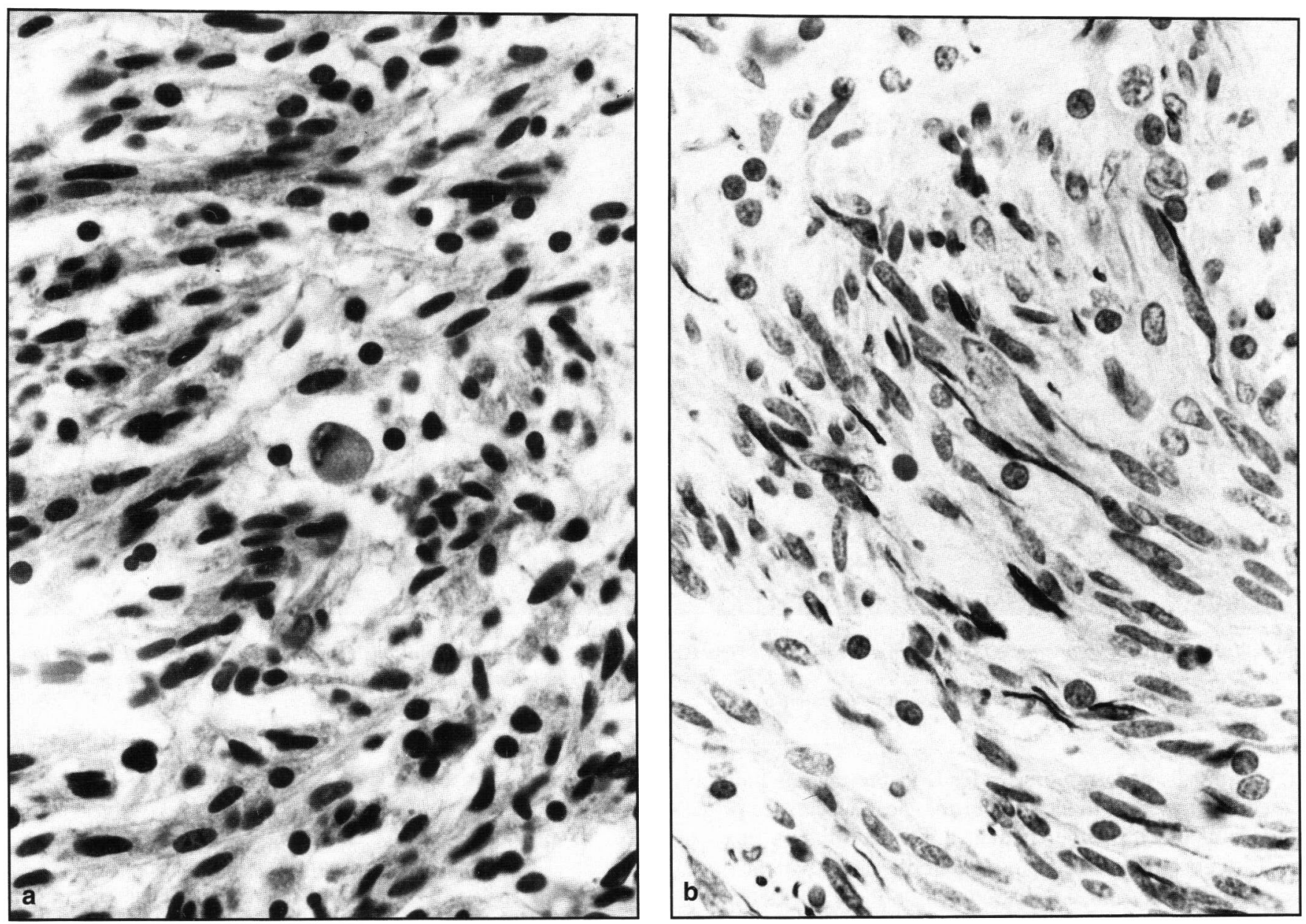

Figure 3: a) Photomicrograph of tumor specimen taken at autopsy. Note that the tumor cells are predominately spindle shaped although rare rounded cells are also found reminiscent of the neoplasm at the time of resection (hematoxylin and eosin $x$ 560). b) Glial fibrillary acidic protein immunohistochemistry (GFAP) shows strong labelling of many of the cell pmocesses (Dako polyclonal GFAP avidin-biotin conjugate technique with aminoethyl carbazole as chromogen $\times 560$ ).

vimentin-positive inclusions quite typical of MRT. Following chemoradiation, however, the residual intracranial tumor cells had a spindle-cell appearance indicative of treatment with retention of the rhabdoid features in the tumor in the spinal cord and meninges. Other tumors such as rhabdomyosarcoma undergo morphological maturation after treatment and it is possible that this maturation may provide a clue to the histogenesis of MRT of brain. Olson ${ }^{18}$ reported three cases of disseminated CNS MRT successfully treated (to date) with a combination of surgery, radiotherapy, and chemotherapy similar to the Intergroup Rhabdomyosarcoma Study III guidelines with vincristine, cisplatin, doxorubicin, cyclophosphamide, actinomycin-D and triple intrathecal chemotherapy (methotrexate, cytosine arabinoside and hydrocortisone), results supporting the theory that CNS MRT is a form of cerebral sarcoma. The lack of GFAP positivity argues against a glial origin but strong S-100 labelling could suggest a neural crest or neuroectodermal derivation or a cartilaginous origin. Vimentin immunopositivity is a mesenchymal feature but the findings of neuroectodermal derivation of the tumor are in line with its appearance in the brain. Whether a similar genotype to that present in the renal rhabdoid tumor is also found in MRT of brain is speculation.
The rapidly lethal outcome of these tumors despite aggressive combined modality therapy with surgery, radiation and chemotherapy is typical of cases which have been reported to date. Whether treatment played a role in the metamorphosis of cellular appearance from polygonal to a spindle cell pattern in Patient 2 is difficult to determine since the cells within the meninges and subarachnoid space remained unaltered. It is possible that the chemotherapy had some effect on the primary tumor by virtue of an impaired blood-brain barrier but the drugs may not have been well distributed in the cerebrospinal fluid. This may indicate that intrathecal chemotherapy may be required as Olson's report seems to indicate. ${ }^{18}$ Certainly a timeconsuming comprehensive metastatic workup is not required because of the systemic nature of this malignancy and the urgent need to initiate therapy.

Bouffe ${ }^{34}$ described a patient with leptomeningeal metastases from MRT who responded to chemotherapy with high dose etoposide and cisplatin. Muller" reported a patient treated with the same type of systemic regimen as was one of our patients, i.e., with ICE chemotherapy (ifosphamide, carboplatin and etoposide). In both cases this chemotherapeutic regimen was relatively ineffective. Agranovich ${ }^{8}$ suggested that craniospinal radiation 

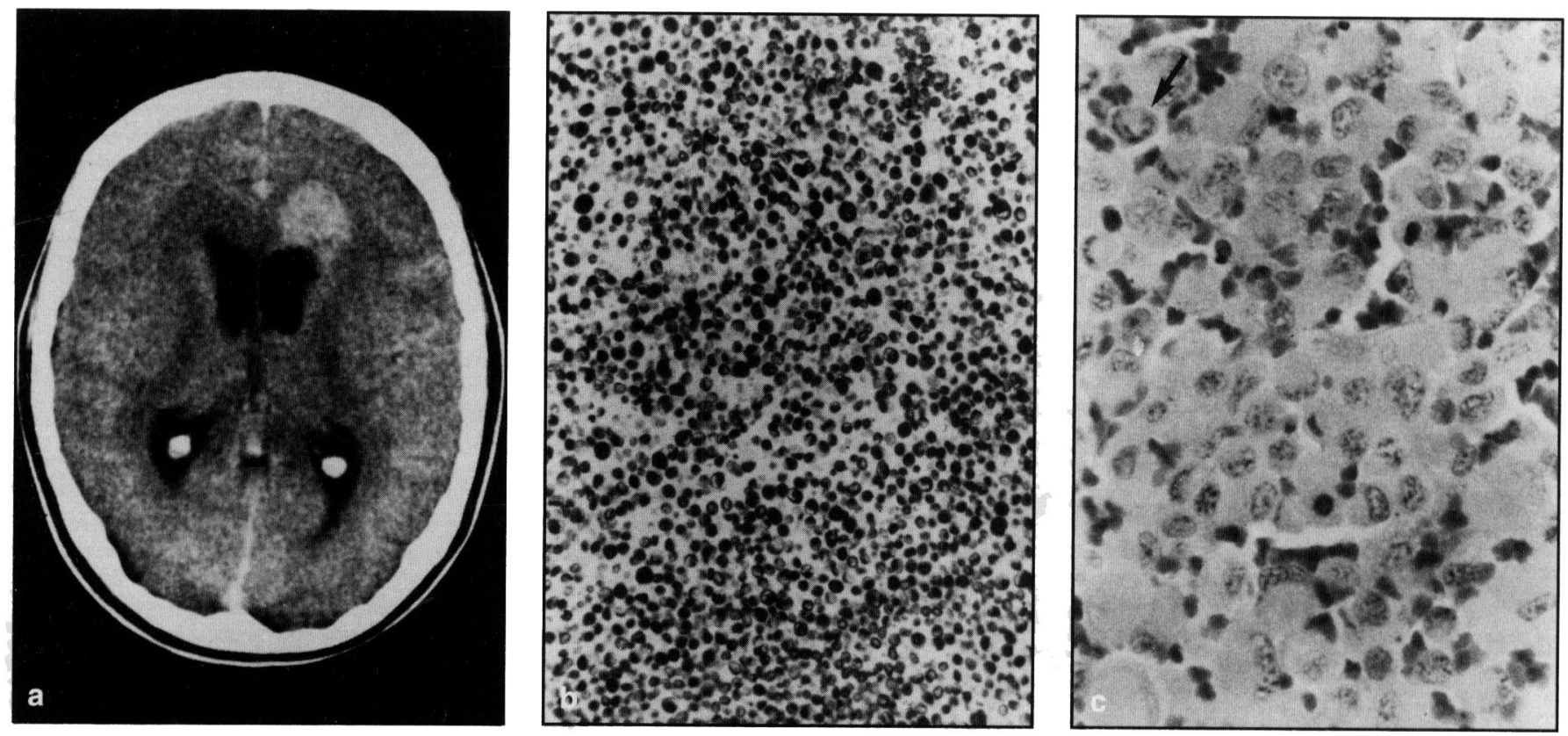

Figure 4: Patient 3. a) Preoperative CT of brain. b) Hematoxylin and eosin $x 240$. Sheets of epithelioid cells with plump nuclei, often displaced to the periphery by the presence of cytoplasmic masses. An example is indicated by arrows. $c$ ) Vimentin immunostain, hematoxylin counterstain $x$ 50. Virtually all tumor cells express intense vimentin immunoreactivity in the cytoplasm. The nucleus is unstained.

might control these tumors. Indeed, in one of our patients (Patient 2) it seemed to alter the histological appearance of the tumor cells but in neither patient did radiation significantly inhibit tumor growth. Perilongo ${ }^{13}$ described two patients, one treated with craniospinal radiation and "8-in-1" chemotherapy who was alive at five months.

A number of conclusions can be drawn from the above cases. First of all, although malignant rhabdoid tumors of brain usually occur in young children, our cases demonstrate that these tumors can occur in all age groups, including adults. The second conclusion which can be drawn involves the histological evolution of tumor cells in one of patients from the typical pretreatment round cell pattern towards a spindle cell pattern post-treatment. Such a histological change would support the theory that these tumors are a type of cerebral sarcoma and therefore would support the treatment approach of Olson who successfully treated three patients with surgical resection, craniospinal radiotherapy and systemic and intrathecal chemotherapy similar to that used for rhabdomyosarcoma. ${ }^{18}$ Lastly, because of the high potential for rapid hematological dissemination this disease should be regarded as systemic in nature and treatment, particularly chemotherapy, shold be initiated promptly after diagnosis. Cerebrospinal fluid cytology is frequently positive and therefore the chemotherapy regimen should include drugs which penetrated the CSF barrier or which are administered intrathecally. Since chemotherapy with traditional agents has been ineffective in this disease, trials of new phase I/II agents are encouraged.

\section{ACKNOWLEDGEMENTS}

The authors thank Mrs. Mary-Ellen Coughlin for typing the manuscript.

\section{REFERENCES}

1. Briner J, Bannwart F, Kleihues P, et al. Malignant small cell tumor of the brain with intermediate filaments - a case of a primary cerebral rhabdoid tumor. Paediatr Pathol 1985; 3 (abst): 117-118.

2. Sotelo-Avila C, Gonzales-Crussi F, deMello D, et al. Renal and extrarenal rhabdoid tumors in children: a clinicopathologic study of 14 patients. Semin Diagn Pathol 1986; 3: 151-163.

3. Kapur S, Patterson K. Primary rhabdoid tumor of the cerebellum Paediatr Pathol 1986; 5: 110.

4. Biggs PJ, Garen PD, Powers, Garvin AJ. Malignant rhabdoid tumor of the central nervous system. Hum Pathol 1987; 18: 332-337.

5. Jakate SM, Marsden HB, Ingram L. Primary malignant rhabdoid tumor of the brain: CT characteristics. Virchows Arch (A) 1988; 412: 393-397.

6. Ho PSP, Lee WH, Chen CY, et al. Primary malignant rhabdoid tumor of the brain: CT characteristics. J Comput Assist Tomogr 1990; 14: 461-463.

7. Chou SM, Anderson JS. Primary CNS malignant rhabdoid tumor (MRT): report of two cases and review of literature. Clin Neuropathol 1991; 10: 1-10.

8. Agranovich AL, Ang LC, Griebel RW, et al. Malignant rhabdoid tumor of the central nervous system with subarachnoid dissemination. Surg Neurol 1992; 37: 410-414.

9. Hanna SL, Langston JW, Parham DM, Douglas EC. Primary malignant rhabdoid tumor of the brain: clinical, imaging, and pathologic findings. Am J Neuroradiol 1993; 14: 107-115.

10. Velasco NM, Brown JA, Kini J, Ruppert ES. Primary congenital rhabdoid tumor of the brain with neoplastic hydranencephaly. Child's Nerv Syst 1993; 9: 185-190.

11. Muller M, Hubbard SL, Provias J, et al. Malignant rhabdoid tumor of the pineal region. Can J Neurol Sci 1994; 21: 273-277.

12. Weeks DA, Malott RL, Zuppan CW, Liwnicz BH, Beckwith JB Primitive cerebral tumor with rhabdoid feature: a case of phenotypic rhabdoid tumor of the central nervous system. Ultrastruct Pathol 1994; 18: 23-28.

13. Perilongo G, Sutton K, Czaykowski D, Gusnard D, Biegel J. Rhabdoid tumor of the central nervous system. Med Pediatr Oncol 1991; 19: 310-317. 
14. Cossu A, Massarelli G, Manetto V, et al. Rhabdoid tumors of the central nervous system: report of three cases with immunocytochemical and ultrastructural findings. Virchows Archiv A Pathol Anat 1993; 422: 81-85.

15. Satoh H, Gioshi J, Sogabe T, et al. Primary malignant rhabdoid tumor of the central nervous system case report and review of the literature. Surg Neurol 1993; 40: 429-430.

16. Bouffet E, Frappaz D, Dolbeau D, et al. Successful treatment for metastatic supratentorial malignant rhabdoid tumor. J Neurooncol 1993; 17: 65-70.

17. Horn M, Schlote W, Lerch K, et al. Malignant rhabdoid tumor: primary intracranial manifestation in an adult. Acta Neuropathol 1992; 83: 445-448.

18. Olson T, Bayar E, Kosnik M, et al. Successful treatment of disseminated central nervous system malignant rhabdoid tumor. J Ped Hematol Oncol 1995; 17: 71-77.

19. Gonzalez-Cruss F, Goldschmidt R, Hseuh W, et al. Infantile sarcoma with intracytoplasmic filamentous inclusions: distinctive tumor of possible histiocytic origin. Cancer 1981; 49: 2365.

20. Beckwith JB, Palmer NF. Histopathology and prognosis of Wilms' tumor. Cancer 1978; 41: 1937-1948.

21. Tsuneyoshi M, Daimaru T, Hashimoto $H$. Malignant soft tissue neoplasms with the histologic features of renal rhabdoid tumors: an ultrastructural and immunohistochemical study. Human Pathology 1985; 16: 1235.

22. Parham DM, Peiper SC, Rabincheaux G. Malignant rhabdoid tumor of the liver: evidence for epithelial differentiation. Arch Pathol Lab Med 1988; 112: 61-64.

23. Lynch HT, Skurin SB, Dahms BB. Paravertebral malignant thabdoid tumor in infancy: in vitro studies of a familial tumor. Cancer 1983; 52: 290-296.
24. Tsokos M, Gregory K, Ramo SC. Malignant rhabdoid tumor of the kidney and soft tissues. Arch Pathol Lab Med 1989; 113: 115120.

25. Haas J, Palmer N, Weinberg A, Beckwith J. Ultrastructure of malignant rhabdoid tumor of the kidney. Hum Pathol 1983; 12: 481-492.

26. Molenaar WM, Jansson DS, Gould VE, et al. Molecular markers of primitive neuroectodermal tumors and other central nervous system tumors. Lab Invest 1989; 61: 635-643.

27. Ota S, Crebbe DC, Tran TN, Traich TJ, Shimada H. Malignant rhabdoid tumor. A study of two established cell lines. Cancer 1993; 71: 2862-2872.

28. Bonnin JM, Rubenstein LT, Palmer NF, Beckwirth JB. The association of embryonal tumors originating in the kidney and brain: a report of seven cases. Cancer 1984; 54: 2137-2146.

29. Biegel JA, Rorke LB, Packer RJ, Emanuel BS. Monosomy 22 in rhabdoid or atypical tumors of the brain. J Neurosurg 1990; 73: 710-714.

30. Dowdy SF, Fasching CL, Araujo D, et al. Suppression of tumorgeneicity in Wilms' tumor by the p15.5-p14 region of chromosome 11. Science 1991; 254-293.

31. Garvin AJ, Regg, Tamowski BI, Hazen-Martin DJ, Sens DA. The G401 cell line, utilized for studies of chromosomal changes in Wilms' tumor, is derived from a rhabdoid tumor of the kidney. Am J Pathol 1993; 142: 375-380.

32. Bouffet E, Franpez D, Dalbeau D, et al. Successful treatment for a metastatic supratentorial malignant rhabdoid tumor. J Neurooncol 1993; 17: 65-70. 